\title{
Determination of the guardbanding to ensure acceptable risk decision in the declaration of conformity
}

\author{
Hicham Mezouara $^{1, \star}$, Latifa Dlimi ${ }^{1}$, Abdelouahhab Salih $^{2}$, and Mohamed Afechcar ${ }^{3}$ \\ 1 Faculty of Science Department of Physics Ibn Tofail University Kenitra, Morocco \\ 2 PES Génie Mécanique LMD- E.N.S.E.T., B.P. 6207 Rabat, Morocco \\ 3 National Center for Studies and Road Research CNER/DRCR BP 6226, Rabat-Instituts Rabat, Morocco
}

Received: 8 May 2014 / Accepted: 12 January 2015

\begin{abstract}
This article proposes a mathematical procedure designed to generate an economical measurement model for determining the risk of decision error (customer risk). The model includes the guardbanding to reduce the impacts of measurement errors. A mathematical model is provided as an example, and conclusions are drawn.
\end{abstract}

Keywords: Guardbanding, consumer risk, measurement errors

\section{Introduction}

In many manufacturing industries, measurement procedures associated with the inspection of products have become an integral part of quality improvement and control. Even so, some measurement errors are inevitable due to changes in operators and/or devices, regardless of how carefully the measurement procedures are designed or maintained. There have been many research efforts to reduce the impact of measurement errors and to improve quality control. The most immediate approach may be to control measurement error by the selection of the guardbanding [1-3]. This paper proposes the scheme for the design of the most economical measurement procedures to determine the guardbanding to reduce the impacts of measurement errors. The remainder of this paper is organized as follows: the effects of guardbanding and precision level on measurement errors are examined and the model for economic design of measurement procedures in terms of the selection of guardbanding and precision level is then proposed and demonstrated through a numerical example. Conclusions are drawn in the last section.

\section{Determination of guardbanding width}

Measurement precision may be improved by reducing measurement variability. Chandra and schall [4] proposed the use of repeated measurements to reduce measurement variability, the average of these repeated measurements is used to determine the conformance of a product to the specifications.

\footnotetext{
* Correspondence: hicham_dess@yahoo.fr
}

Let $X$ be the actual value of the quality characteristic of interest, which is normally distributed with a mean of $\mu$ and a variance of $\sigma_{X}^{2}$. If we denote the measured value from a single measurement as $Y$, let us further assume that the conditional distribution of $Y$, given that $X=x$, is a normal distribution with a mean of $x$ and a variance $\sigma_{y \mid x}^{2}$.

Suppose that $n$ measurements are repeatedly taken and each measurement has the same variability. Letting $\bar{Y}$ be the average of $n$ measurements, it is apparent $\sigma_{\bar{y} \mid x}^{2}$, where $\sigma_{\bar{y} \mid x}^{2}=\frac{\sigma_{y \mid x}^{2}}{n}$. As a means of reducing the impact of measurement errors, the use of guard bands has been widely implemented since being introduced by Eagle [5].

In many practical situations, a false acceptance of defects incurs much larger economic penalties than a false rejection of conforming products. From this perspective, many manufacturers impose a guardbanding to help minimize the penalty associated with false acceptance, at the cost of an increased risk of false rejection. The effects of a guardbanding are depicted in that the conditional distribution of $\bar{Y}$, given that $X=x$, is a normal distribution with a mean of $x$ and a variance of Figure 1 , where $L$ and $U$ represent the lower and upper specification limits. The large curve represents the density curve of the actual value of the quality characteristic, $X$, while the small curve represents the density curve of the average measurements given the actual value, $(\bar{Y} \mid X)$. It can be observed that the probability of false acceptance decreases by imposing the guardbanding (Fig. 1a) while the risk associated with false rejection increases (Fig. 1b). It is a current practice to set the guardbanding based on engineering experiences or on a trial-and-error basis. 

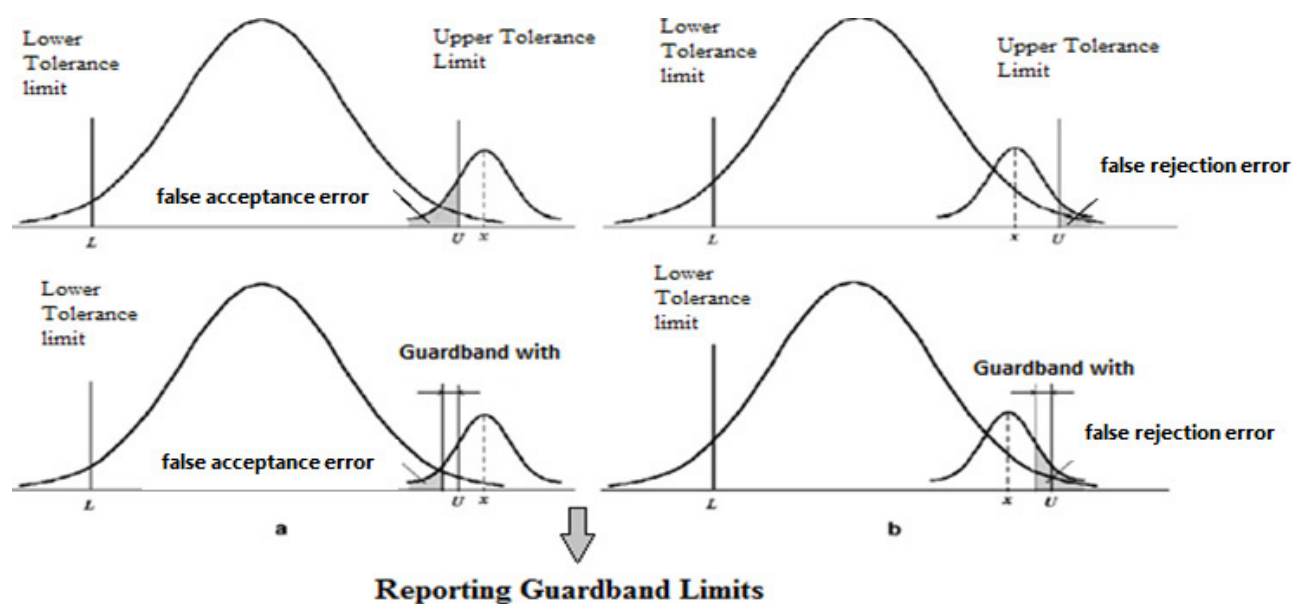

Reporting Guardband Limits

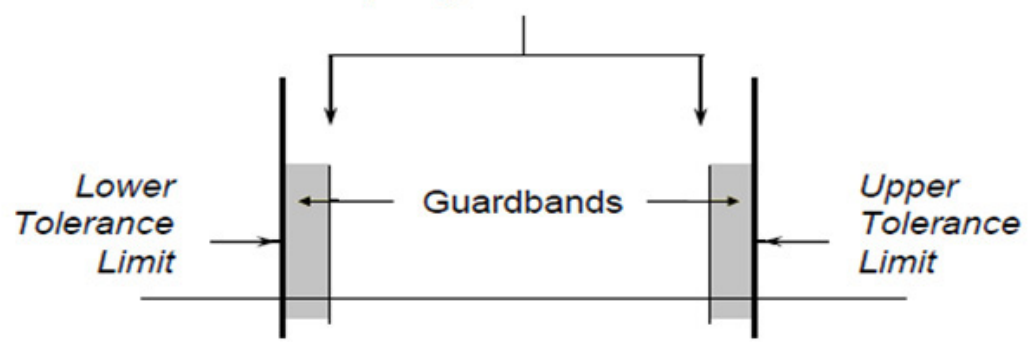

Fig. 1. Measurement errors with and without guard band. (a) False acceptance error with and without guard band. (b) False rejection error with and without guard band.

To meet customer requirements and to avoid the high cost of passing bad product to customers, the consumer's risk should not exceed a specified value. In this case, the guardbanding $v$ and $\omega$ are set inside the specification limits $L$ and $U$. Let $v=L+\varepsilon_{L}$ and $\omega=U-\varepsilon_{U}$, then $\varepsilon_{L}$ and $\varepsilon_{U}$ are positive. On the other hand, if the consumer's risk exceeds the specified value, then $\varepsilon_{L}$ or $\varepsilon_{U}$ may be negative. In this paper, we focus on meeting customer's requirements so that $\varepsilon_{L}$ and $\varepsilon_{U}$ are positive. In general, the consumer's risk should be much lower than the producer's risk because the cost of letting bad product get to consumers is usually much higher than the cost of rejecting good product. The difference between the product tolerance and the length of the guardbanding interval is $(U-L)-(v-\omega)=\left(\varepsilon_{L}+\varepsilon_{U}\right)$. The optimal guardbanding interval $(v, \omega)$ or the pair $\left(\varepsilon_{L}, \varepsilon_{U}\right)$ with the smallest $\left(\varepsilon_{L}+\varepsilon_{U}\right)$ can be determined so that $\beta \leqslant \beta_{0}$ where $\beta_{0}$ preset level and the expression for $\beta$ are given by equation (20).

\section{Study of customer risk}

The customer risk is the percentage of non-conforming products that are delivered, and accepted by the customer. it is calculated as the product of the probability of making a "non-conforming" product (property of the production process) by the (conditional) probability of measuring "compliant" (i.e. in the tolerance).

Let $\varepsilon_{L}$ and $\varepsilon_{U}$ denote the widths of guardbanding associated with the lower and upper specification limits, respectively. For the simplicity of notation, $v=L+\varepsilon_{L}$ and
$\omega=U-\varepsilon_{U}$. Hereafter, $v$ and $\omega$ are referred to as the lower and upper inspection limits, respectively. Since the conformance of a product is determined on the basis of repeated measurements, a product passes the inspection and is shipped to the customer if $\bar{y} \epsilon[v, \omega]$ and $R_{C}=\beta$ represent the customer risk (the risk of delivering a product that is intrinsically unacceptable but that is accepted by the control means), i.e., $\bar{y} \in[v, \omega]$ and $X[L, U]$.

The expected cost by falsely accepting a defect, denoted by $\beta^{\prime}$, is the conditional probability that a product will be accepted given that it is defective. it is then given by:

$$
\beta^{\prime}=\frac{\beta}{1-P(X \in[L, U])},
$$

where $P(x \in[L, U])$ is the probability that the value being measured lies in $[L, U]$.

$$
\begin{aligned}
R_{C}=\beta= & \int_{v}^{\omega} \int_{U}^{+\infty} h(x, \bar{y}) d x d \bar{y} \\
& +\int_{v}^{\omega} \int_{-\infty}^{L} h(x, \bar{y}) d x d \bar{y},
\end{aligned}
$$

where $h(x, \bar{y})$ is the joint density function of $X$ and $\bar{Y}$. It can easily be shown that $X$ and $\bar{Y}$ jointly follow a bivariate normal distribution with a mean vector of $(\mu, \mu)$ and a variance-covariance matrix of $\Sigma$ given by:

$$
\Sigma=\left[\begin{array}{cc}
\operatorname{var}(x) & \operatorname{cov}(x, \bar{y}) \\
\operatorname{cov}(x, \bar{y}) & \operatorname{var}(\bar{y})
\end{array}\right]=\left[\begin{array}{cc}
\sigma_{x}^{2} & \sigma_{x}^{2} \\
\sigma_{x}^{2} & \sigma_{\bar{y}}^{2}
\end{array}\right]
$$


where $\sigma_{\bar{y}}^{2}$ represents the variance of the marginal distribution of $\frac{y}{Y}$, and $\sigma_{\bar{y}}^{2}=\sigma_{x}^{2}+\sigma_{\bar{y} \mid x}^{2}$. Note that $\gamma$, the correlation coefficient of $X$ and $\bar{Y}$, is defined as:

$$
\gamma=\frac{\operatorname{cov}(X, \bar{Y})}{\sqrt{\operatorname{var}(X) \times \operatorname{var}(\bar{Y})}}=\frac{\sigma_{x}}{\sigma_{\bar{y}}} .
$$

The marginal distribution $X$ and $\bar{Y}$, assume that the density $h(x, \bar{y})$ exists, we denote by $g(\bar{y} \mid x)$ and $f(x)[6-8]$.

And

$$
h(x, \bar{y})=g(\bar{y} \mid x) f(x),
$$

where

$$
g(\bar{y} \mid x)=\frac{1}{\sqrt{2 \pi} \sigma_{\bar{y} \mid x}} e^{-\frac{(\bar{y}-x)^{2}}{2 \sigma_{\bar{y}} \mid x}}
$$

and

$$
f(x)=\frac{1}{\sqrt{2 \pi} \sigma x} e^{-\frac{(x-\mu)^{2}}{2 \sigma_{x}^{2}}}
$$

The first double integral in equation (2) can be written as:

$$
\begin{aligned}
\int_{v}^{\omega} \int_{U}^{\infty} h(x, \bar{y}) d x d \bar{y} & =\int_{U}^{\infty} \int_{v}^{\omega} h(x, \bar{y}) d \bar{y} d x \\
& =\int_{U}^{\infty}\left[\int_{v}^{\omega} g(\bar{y} \mid x) d \bar{y}\right] f(x) d x
\end{aligned}
$$

where $g(\bar{y} \mid x)$ and $f(x)$ are the conditional distribution of $\bar{Y}$ since $X=x$ and the marginal distribution of $X$, respectively. Noting that

$$
\left.\bar{Y}\right|_{X=x} \approx N\left(x, \sigma_{\bar{y} \mid x}^{2}\right)
$$

and

$$
X \approx N\left(\mu, \sigma_{x}^{2}\right) .
$$

Let $z=\frac{\bar{y}-x}{\sigma_{\bar{y} \mid x}}$, and $v \leqslant \bar{y} \leqslant \omega$ is then given

$$
\frac{v-x}{\sigma_{\bar{y} \mid x}} \leqslant z \leqslant \frac{\omega-x}{\sigma_{\bar{y} \mid x}} .
$$

And

$$
\lambda=\frac{x-\mu}{\sigma_{x}},
$$

with $U \leqslant x \leqslant+\infty$ is then given

$$
\frac{U-\mu}{\sigma_{\mathrm{x}}} \leqslant \lambda \leqslant+\infty \text {. }
$$

Can be written as equation (8):

$$
\begin{aligned}
\int_{U}^{\infty} & {\left.\left[\int_{v}^{\omega} g(\bar{y} \mid x) d \bar{y}\right] f(x) d x=\int_{U}^{\infty} \int_{\left[\frac{v-x}{\sigma \bar{y} \mid x}\right.}^{\frac{\omega-x}{\sigma_{\bar{y} \mid x}}} \phi(z) d z\right] f(x) d x } \\
= & \int_{U}^{\infty}\left[\Phi\left(\frac{\omega-x}{\sigma_{\bar{y} \mid x}}\right)-\Phi\left(\frac{v-x}{\sigma_{\bar{y} \mid x}}\right)\right] f(x) d x \\
= & \int_{U}^{\infty} \Phi\left(\frac{\omega-x}{\sigma_{\bar{y} \mid x}}\right) f(x) d x-\int_{U}^{\infty} \Phi\left(\frac{\nu-x}{\sigma_{\bar{y} \mid x}}\right) f(x) d x \\
= & \int_{U-\mu}^{\infty} \Phi\left(\frac{\omega-\left(\mu+\sigma_{x} \lambda\right)}{\sigma_{\bar{y} \mid x}}\right) \phi(\lambda) d \lambda \\
& -\int_{U-\mu / \sigma_{x}}^{\infty} \Phi\left(\frac{\nu-\left(\mu+\sigma_{x} \lambda\right)}{\sigma_{\bar{y} \mid x}}\right) \phi(\lambda) d \lambda .
\end{aligned}
$$

Here, $\Phi($.$) and \varphi($.$) represent the cumulative distribution$ and probability density functions of the standard normal distribution, respectively [9]. Using the following identity,

$$
\int_{K}^{\infty} \Phi(a+b \lambda) \phi(\lambda) d \lambda=B V N\left(\frac{a}{\sqrt{1+b^{2}}} ;-K ; \frac{b}{\sqrt{1+b^{2}}}\right)
$$

where $B V N(\alpha, \beta, \rho)$ represents a function with two variables standard normal distribution with a correlation coefficient of $\rho$, which is defined by:

$$
\begin{aligned}
B V N(\alpha, \beta, \rho)= & \frac{1}{2 \pi \sqrt{1-\rho^{2}}} \\
& \times \int_{-\infty}^{\alpha} \int_{-\infty}^{\beta} \exp \left(-\frac{x^{2}-2 \rho x y+y^{2}}{2\left(1-\rho^{2}\right)}\right) d x d y
\end{aligned}
$$

with

$$
\begin{aligned}
& \varphi(x)=\frac{d \Phi(x)}{d x} \\
& \Phi(x)=\int_{-\infty}^{h} \varphi(x) d x
\end{aligned}
$$

and

$$
\varphi(x)=\frac{1}{\sqrt{2 \pi}} \exp \left(-\frac{x^{2}}{2}\right) .
$$


Equation (12) can be simplified to

$$
\begin{aligned}
\int_{v}^{\omega} \int_{U}^{\infty} h(x, \bar{y}) d x d \bar{y}= & B V N\left(\frac{\omega-\mu}{\sqrt{\sigma_{x}^{2}+\sigma_{\bar{y} \mid x}^{2}}} ;-\frac{U-\mu}{\sigma_{x}} ;-\gamma\right) \\
& -B V N\left(\frac{v-\mu}{\sqrt{\sigma_{x}^{2}+\sigma_{\bar{y} \mid x}^{2}}} ;-\frac{U-\mu}{\sigma_{x}} ;-\gamma\right),
\end{aligned}
$$

Similarly, it can be shown that the second integral in equation (2) becomes:

$$
\begin{aligned}
\int_{v}^{\omega} \int_{-\infty}^{L} h(x, \bar{y}) d x d \bar{y}= & \Phi\left(\frac{\omega-\mu}{\sqrt{\sigma_{x}^{2}+\sigma_{\bar{y} \mid x}^{2}}}\right)-\Phi\left(\frac{v-\mu}{\sqrt{\sigma_{x}^{2}+\sigma_{\bar{y} \mid x}^{2}}}\right) \\
& +B V N\left(\frac{v-\mu}{\sqrt{\sigma_{x}^{2}+\sigma_{\bar{y} \mid x}^{2}}} ;-\frac{L-\mu}{\sigma_{x}} ;-\gamma\right) \\
& -B V N\left(\frac{\omega-\mu}{\sqrt{\sigma_{x}^{2}+\sigma_{\bar{y} \mid x}^{2}}} ;-\frac{L-\mu}{\sigma_{x}} ;-\gamma\right) .
\end{aligned}
$$

Using equations (18) and (19), the customer risk $R c$ can be written as:

$$
\begin{aligned}
R c=\beta= & \Phi\left(\frac{\omega-\mu}{\sigma_{\bar{y}}}\right)-\Phi\left(\frac{v-\mu}{\sigma_{\bar{y}}}\right) \\
& +B V N\left(\frac{\omega-\mu}{\sigma_{\bar{y}}} ;-\frac{U-\mu}{\sigma_{x}} ;-\gamma\right) \\
& -B V N\left(\frac{\omega-\mu}{\sigma_{\bar{y}}} ;-\frac{L-\mu}{\sigma_{x}} ;-\gamma\right) \\
& -B V N\left(\frac{v-\mu}{\sigma_{\bar{y}}} ;-\frac{U-\mu}{\sigma_{x}} ;-\gamma\right) \\
& +B V N\left(\frac{v-\mu}{\sigma_{\bar{y}}} ;-\frac{L-\mu}{\sigma_{x}} ;-\gamma\right) .
\end{aligned}
$$

\section{A numerical example}

To demonstrate the proposed model, consider an example of indirect tensile tests of stiffness modulus, according to standard NF EN 12697-26:2004 [10], tests on cylindrical specimens $100 \mathrm{~mm}$ in diameter, with thickness of $53 \mathrm{~mm}$ and $2451 \mathrm{~kg} / \mathrm{m}^{2}$ density, were carried out under the conditions listed in Table 1.

We obtained a Stiffness modulus $\bar{E}^{*}=6696 \mathrm{MPa}$, with an estimated standard deviation $(\sigma)$ of $382.50 \mathrm{MPa}$ with $\bar{E}^{*}=\frac{\sum E_{i}^{*}}{10}, n=10$ (the average shear modulus corrected). The uncertainty has been calculated by the testing laboratory using an analytical method based on [11]. The requirements agreed upon between the customer and the supplier specified a lower specification limit, $L$, of $6000 \mathrm{MPa}$, and an upper specification limit, $U$, of $10000 \mathrm{MPa}$.
Table 1. Conditions of the test of the proposed model.

\begin{tabular}{cc}
\hline Horizontal deformation under & $5 \pm 2 \mu \mathrm{m}$ \\
\hline Frequency & $10 \mathrm{~Hz}$ \\
Number of pulses & 10 \\
The pulse repetition period & $3 \pm 0.1 \mathrm{~s}$ \\
Rise time in load & $124 \pm 4 \mathrm{~ms}$ \\
Poisson's ratio & 0.35 \\
\hline
\end{tabular}

Table 2. Numerical results of the customer risk of the stiffness modulus.

\begin{tabular}{cccccc}
\hline$\varepsilon_{L} / \varepsilon_{U}$ & 0.00 & 0.25 & 0.50 & 0.75 & 1.00 \\
\hline 0.00 & 0.70665 & 0.70669 & 0.70675 & 0.70679 & 0.70684 \\
0.25 & 0.70660 & 0.70664 & 0.70669 & 0.70674 & 0.70678 \\
0.50 & 0.70654 & 0.70654 & 0.70664 & 0.70668 & 0.70673 \\
0.75 & 0.70649 & 0.70653 & 0.70658 & 0.70663 & 0.70668 \\
1.00 & 0.70644 & 0.70648 & 0.70653 & 0.70658 & 0.70662 \\
\hline
\end{tabular}

The supplier has taken ten cylindrical specimens $(n=$ 10) for the control, the variability of the 10 measures is given by:

$$
\sigma_{y \mid x}=\sqrt{\frac{\sum\left(n_{i}-\bar{x}\right)^{2}}{n-1}}=296 \mathrm{MPa}
$$

with

$$
\begin{aligned}
& \sigma_{\bar{y}}^{2}=\sigma_{x}^{2}+\sigma_{\bar{y} \mid x}^{2} \\
& \sigma_{\bar{y}}=\sqrt{\sigma_{x}^{2}+\sigma_{\bar{y} \mid x}^{2}}=\sqrt{\sigma_{x}^{2}+\sigma_{\frac{y \mid x}{n}}^{2}}=393.78 \mathrm{MPa}
\end{aligned}
$$

and

$$
\gamma=\frac{\operatorname{cov}(X, \bar{Y})}{\sqrt{\operatorname{var}(X) \cdot \operatorname{var}(\bar{Y})}}=\frac{\sigma_{x}}{\sigma_{\bar{y}}}=0.97 .
$$

Solving the mathematical model requires a lot of computing resources mainly due to the evaluation of bivariate normal probabilities. However, an approximation algorithm, developed with the free software $\mathrm{R}$ programming language [12], was utilized to evaluate the integrals bivariate normal.

We suppose that $\beta_{0}=0.70648 \%$ the optimal solution to the example problem is found to be $v^{*}=6000 \mathrm{MPa}$, $\omega^{*}=9999 \mathrm{MPa}, \varepsilon_{L}=1.00 \mathrm{MPa}$ and $\varepsilon_{U}=0.00 \mathrm{MPa}$, $n=10$, with acustomer risk of $0.70644 \%$, guardbanding provide a way of assuring that good product would be accepted $99.23 \%$. Numerical results are summarized in Table 2.

\section{Conclusion}

In many industrial settings, manufacturing processes often involve inspection procedures which frequently require measurement of a quality characteristic. Various economic aspects of measurement errors have long been studied. Further, there has been a great deal of research efforts to reduce the impacts of errors. This paper proposes the 
model for economic design of measurement systems by incorporating the concepts of measurement precision and guardbanding.

The proposed model incorporates various economic aspects of measurement errors when determining the level of measurement precision and widths of guardbanding to minimize the impacts of measurement errors. A numerical example demonstrates the proposed model.

\section{References}

1. D. McCarville, D. Montgomery, Optimal guard bands for gauges in series, Quality Eng. 9, 167-177 (1996)

2. B. Hutchinson, Setting guardband test limits to satisfy MIL-STD-45662A requirements, Proc. of 1991 NCSL Workshop and Symposium (1991), pp. 305-309

3. D. Deaver, Using Guardbands to Justify TURs Less than 4:1, Proc. of ASQC 49th Annual Quality Congress (1995), pp. 136-141
4. J. Chandra, S. Schall, The use of repeated measurements to reduce the effect of measurement errors, IIE Trans. 20, 83-87 (1988)

5. A. Eagle, A method for handling errors in testing and measuring, Industrial Quality Control 10(5), 10-15 (1954)

6. J. Brucker, A note on the bivariate normal distribution, Commun. Statist A 8, 175-177 (1979)

7. Fraser DAS, F. Streit, A further note on the bivariate normal distribution, Commun. Statist A 9, 1097-1099 (1980)

8. M. Ahsanullah, Some characterizations of the bivariate normal distribution, Metrika 32, 215-218 (1985)

9. S. Chen, K. Chung, Selection of the optimal precision level and target value for a production process: the lowerspecification limit case, IIE Trans. 28, 979-985 (1996)

10. AFNOR, Méthodes d'essai pour mélange hydrocarboné à chaud : Module de Rigidité, NF EN 12697-26 (2004)

11. AFNOR, Guide pour l'expression de l'incertitude de mesure, NF ENV 13005 (1999)

12. P.Dalgaard, Introductory Statistics with $R$, 2nd edn. (Springer, New York, 2008) 